\title{
Candida albicans biofilm on titanium: effect of peroxidase precoating
}

This article was published in the following Dove Press journal:

Medical Devices: Evidence and Research

31 July 2010

Number of times this article has been viewed

\author{
Mohamed Ahariz' \\ Philippe Courtois ${ }^{1,2}$ \\ 'Laboratory of Experimental \\ Hormonology, Université Libre de \\ Bruxelles, Brussels, ${ }^{2}$ UER de Biologie \\ Médicale, Haute Ecole Francisco \\ Ferrer, Brussels, Belgium
}

\begin{abstract}
The present study aimed to document Candida albicans biofilm development on titanium and its modulation by a peroxidase-precoated material which can generate antimicrobials, such as hypoiodite or hypothiocyanite, from hydrogen peroxide, iodide, or thiocyanate. For this purpose, titanium (powder or foil) was suspended in Sabouraud liquid medium inoculated with C. albicans ATCC10231. After continuous stirring for 2-21 days at room temperature, the supernatant was monitored by turbidimetry at $600 \mathrm{~nm}$ and titanium washed three times in sterile Sabouraud broth. Using the tetrazolium salt MTT-formazan assay, the titanium-adherent fungal biomass was measured as $7.50 \pm 0.60 \times 10^{6}$ blastoconidia per gram of titanium powder $(\mathrm{n}=30)$ and $0.50 \pm 0.04 \times 10^{6}$ blastoconidia per $\mathrm{cm}^{2}$ of titanium foil $(\mathrm{n}=12)$. The presence of yeast on the surface of titanium was confirmed by microscopy both on fresh preparations and after calcofluor white staining. However, in the presence of peroxidase systems (lactoperoxidase with substrates such as hydrogen peroxide donor, iodide, or thiocyanate), Candida growth in both planktonic and attached phases appeared to be inhibited. Moreover, this study demonstrates the possible partition of peroxidase systems between titanium material (peroxidase-precoated) and liquid environment (containing peroxidase substrates) to limit C. albicans biofilm formation.
\end{abstract}

Keywords: adhesion, material, oral, yeast

\section{Introduction}

Yeasts can colonize titanium surfaces even after implantation. Because yeasts form biofilms on titanium surfaces, they can become the source of device-related infections with subsequent morbidity and occasionally death of the patient, all bearing high costs. Yeast colonization is frequently found in catheters and indwelling devices in cancer patients, for whom parenteral nutrition and immunosuppression are aggravating factors. ${ }^{1-4}$ Fungal biofilms are difficult to treat on account of their increased resistance to antimicrobial agents. This is the reason why fungemia associated with catheterization or prosthetic devices should prompt the removal of these implanted materials, because antifungal drug treatment remains an uncertain alternative.,

Prophylactic antibiotic therapy to prevent bacterial device-associated infections occasionally leads to increased mycotic complications. ${ }^{4}$ In dentistry, Candida species and periodontal pathogens have been isolated around titanium implants. ${ }^{7,8}$ Titanium-coated silicone was thought to prevent Candida biofilm formation on voice prostheses by increasing the smoothness of the material surface. ${ }^{9}$ Titanium material does not possess antifungal properties by itself, but $\mathrm{TiO}_{2}$ can act as a photocatalyst for the decomposition of organic compounds under ultraviolet light, thereby reducing the viability of Candida albicans. ${ }^{10,11}$ Bioactive coating, used as an osseointegration
Correspondence: Philippe Courtois Laboratory of Experimental Hormonology (CP 626), Université Libre de Bruxelles (ULB), Route de Lennik 808, B-1070 Brussels, Belgium

$\mathrm{Tel}+3225556310$

Fax +32 25554219

Email philippe.courtois@ulb.ac.be 
facilitator, is another option for controlling biofilm formation. In order to reduce bacterial and fungal infections, some groups have proposed the precoating of orthopedic devices with antimicrobial agents (chlorhexidine/chloroxylenol). ${ }^{12}$ However, bioactive protein coating is not ideal for in vivo use because it can induce adverse immunologic reactions, yet remains an interesting area for studying biofilms in vitro. Adsorption of lactoperoxidase (LP) onto titanium surfaces, which has antimicrobial properties similar to those of oral peroxidase, has already been described. ${ }^{13} \mathrm{LP}$ produces hypothiocyanite $\left(\mathrm{OSCN}^{-}\right)$and hypothiocyanous acid $(\mathrm{HOSCN})$ compounds from thiocyanate $\left(\mathrm{SCN}^{-}\right)$in the presence of hydrogen peroxide $\left(\mathrm{H}_{2} \mathrm{O}_{2}\right)$. In vitro, iodide $\left(\mathrm{I}^{-}\right)$has also been used as substrate, because it is oxidized into hypoiodite $\left(\mathrm{OI}^{-}\right)$ or hypoiodous acid (OIH) by peroxidase activity. ${ }^{14}$

Nevertheless, attempts to understand interface biology better and to improve material surface treatment need a model of biofilm on titanium pieces that are easy to manipulate in the laboratory. Few studies have developed a titanium model in order to investigate procedures for limiting yeast adherence and biofilm formation. ${ }^{15,16}$ The present study aimed at demonstrating and quantifying both $C$. albicans adherence to titanium (powder and foil) and its modulation in the presence of peroxidase systems.

\section{Materials and methods Materials}

Candida adherence to titanium (powder or foil) was evaluated in comparison with its adherence to other materials, such as acrylic resins (hard or soft) and polystyrene, which are commonly used oral biomaterials and laboratory assay tube manufacture materials, respectively. Aliquots $(0.5 \mathrm{~g})$ of titanium powder (Alfa Aesar ${ }^{\circledR}$, Karlsruhe, Germany; 99.5\% purity, $\sim 325$ mesh) were transferred into sterile polystyrene cell culture tubes (BD Falcon ${ }^{\circledR}$, Franklin Lakes, NJ; size $125 \times 16 \mathrm{~mm}$, round bottom). Titanium pieces were cut from native foil (Advent ${ }^{\circledR}$, Oxford; 99.6\% purity; thickness $25 \mu \mathrm{m}$, size $25 \mathrm{~mm} \times 5 \mathrm{~mm}$ ). As previously described, ${ }^{13}$ each piece was weighed and the total surface was calculated using the following algorithm: $\mathrm{S}=2\{(\mathrm{~m} / \mathrm{\rho h})+\mathrm{h}(\mathrm{L}+\mathrm{I})\}$ where $\mathrm{S}=$ total surface $\left(\mathrm{mm}^{2}\right), \mathrm{m}=$ foil mass $(\mathrm{mg}), \rho=$ volumic $\operatorname{mass}\left(4.544 \mathrm{mg} / \mathrm{mm}^{3}\right), \mathrm{h}=$ foil thickness $(0.025 \mathrm{~mm}), \mathrm{L}=$ foil length $(\mathrm{mm}), 1=$ foil width $(\mathrm{mm})$. Total surface averaged $246 \pm 4 \mathrm{~mm}^{2}(\mathrm{n}=16)$ with a variation coefficient of $6.5 \%$.

Two different resins for dentures (Vertex soft ${ }^{\circledR}$ and Vertex Rapid Simplified $^{\circledR}$; Vertex Dental, Zeist, Netherlands) were investigated with and without varnishing (Palaseal ${ }^{\circledR}$; Heraeus
Kulzer, Wehrheim, Germany). Acrylic resin pieces (thickness $\sim 2 \mathrm{~mm}$, size $25 \mathrm{~mm} \times 5 \mathrm{~mm}$ ) were processed according to manufacturer's instructions. The mass of each resin piece averaged $222 \pm 19 \mathrm{mg}( \pm \mathrm{SD}, \mathrm{n}=96)$ with a coefficient of variation of $8.6 \%$.

\section{Cultures of Candida albicans}

C. albicans ATCC10231 (Culti-Loops ${ }^{\circledR}$, Oxoid ${ }^{\circledR}$, Basingstoke, UK) was grown at $37^{\circ} \mathrm{C}$ on Sabouraud-gentamicinchloramphenicol agar (Becton-Dickinson ${ }^{\circledR}$, Belgium). Yeasts were harvested from the third subculture and used to inoculate Sabouraud broth adjusted to an absorbance of 0.800 at $600 \mathrm{~nm}$. The Candida count was conducted in the adjusted suspension after culture $\left(37^{\circ} \mathrm{C}\right.$ for two days) on Sabouraud agar of $20 \mu \mathrm{L}$ aliquots from serial dilutions (until 104-fold) in Sabouraud broth. The number of colony-forming units (CFU) in the initial suspension yielded $16 \pm 3 \times 10^{6} \mathrm{CFU} / \mathrm{mL}(\mathrm{n}=6)$.

\section{Candida albicans adherence}

Titanium powder $(0.5 \mathrm{~g})$ was suspended in $3.6 \mathrm{~mL}$ Sabouraud broth and yeast cells $(0.4 \mathrm{~mL}$ at included concentration) were added. Negative controls consisted of titanium powder incubated in $4 \mathrm{~mL}$ sterile Sabouraud broth. After continuous stirring (three revolutions per minute, Stuart ${ }^{\circledR}$ SB3 rotator, Barloworld Scientific, Stone, UK) for two days at room temperature, the titanium powder was separated from the liquid medium by sedimentation (10 minutes) and washed three times with $4 \mathrm{~mL}$ sterile Sabouraud broth, changing the assay tube for each washing cycle. From some negative controls, after two days of incubation and removal of sterile Sabouraud, titanium powder was contaminated by $4 \mathrm{~mL}$ of the first supernatant from the contaminated assays in order to evaluate washing process efficiency. The Candidal titanium contact time was then reduced to sedimentation time (10 minutes). All the supernatants were monitored by turbidimetry at $600 \mathrm{~nm}$ to evaluate the planktonic yeast phase. Titanium and resin foils were similarly treated. Materialadherent yeast biomass (anchored yeast phase) was evaluated by the tetrazolium salt MTT $(1.7 \mathrm{mg} / \mathrm{mL}$ in $2: 1$ Sabouraud phosphate-buffered solution at $\mathrm{pH}$ 7.40) which is enzymatically reduced by viable fungi, with resultant formation of MTT-formazan absorbing at $570 \mathrm{~nm} \cdot{ }^{17}$ After MTT-formazan extraction with isopropanol, absorbance was immediately measured in a spectrophotometer at $570 \mathrm{~nm}$. The main characteristics of the method were: molar absorption coefficient ${ }^{18} \varepsilon 570$ of $17,000 \mathrm{M}^{-1} \times \mathrm{cm}^{-1}$, sensitivity of $0.5 \times 10^{6}$ blastoconidia $/ \mathrm{mL}$, coefficient of variation $\leq 12.0 \%$, analytic 
range $0.5-10 \times 10^{6}$ blastoconidia $/ \mathrm{mL}$. Figure 1 illustrates the rate of tetrazolium salt MTT reduction into formazan in the presence of a fixed number of blastoconidia as a function of the tetrazolium salt concentration.

\section{Microscopic inspection}

C. albicans adherent to titanium powder were observed using a light microscope (fresh preparation) equipped with epifluorescence (after calcofluor white staining). Yeastcontaminated titanium was examined microscopically in the presence of $1 \mathrm{mg} / \mathrm{mL}$ calcofluor white M2R stain (fluorescent brightener 28; Sigma $^{\circledR}$, St Louis, MO) known as a marker of cell wall chitin.

\section{Lactoperoxidase adsorption on titanium}

LP (Biopole ${ }^{\circledR}$, Belgium) was dissolved in demineralized water. Pieces of titanium foil were immersed for 30 minutes at $37^{\circ} \mathrm{C}$ in $5 \mathrm{~mL}$ of a $0.8 \mathrm{mg} / \mathrm{mL}$ LP solution or in demineralized water alone (control). After incubation, the liquid phase was removed and titanium foils were washed three times
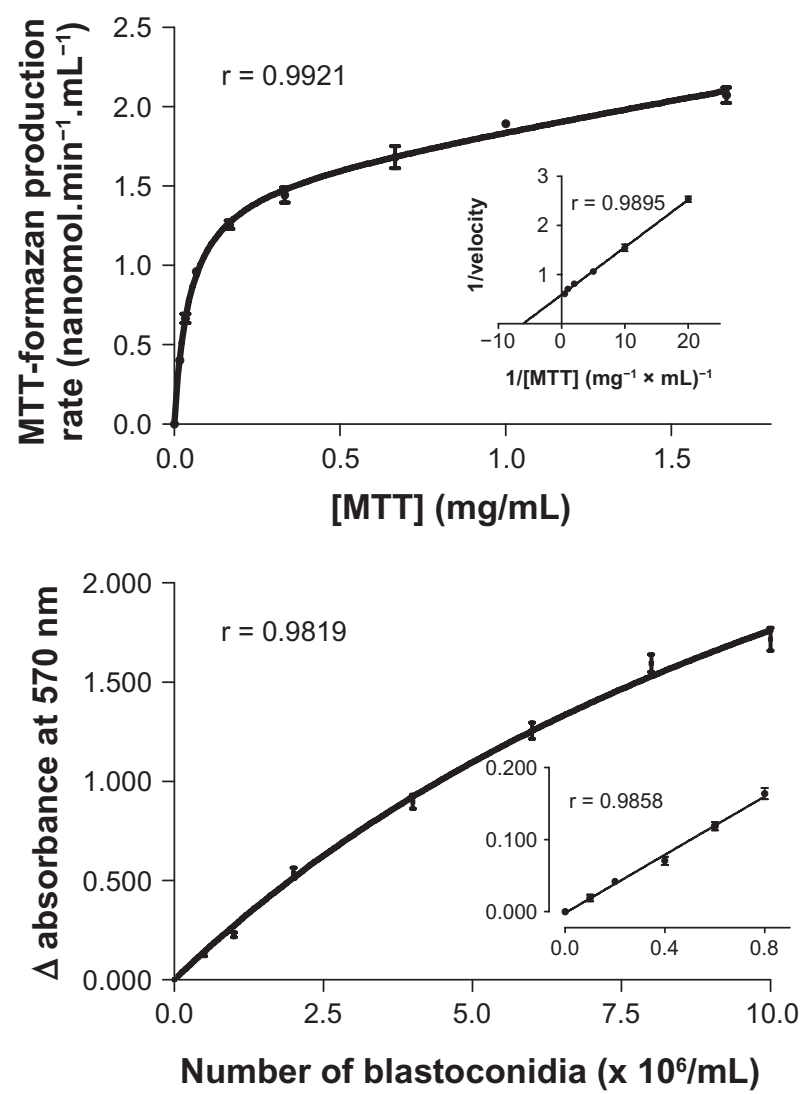

Figure I MTT procedure. Top: Rate of tetrazolium salt MTT reduction as a function of substrate concentration in the presence of a fixed blastoconidia number (Cartesian presentation of data with double reciprocal plot displayed in the insert). Bottom: Reduction of tetrazolium salt MTT as a function of Candida albicans blastoconidia numbers (data for the lowest values are presented in the insert with more appropriate coordinates). with $5 \mathrm{~mL}$ of demineralized water and then transferred into another tube with the last washing. This procedure was repeated five times prior to a last removal of the liquid phase.

\section{Lactoperoxidase systems}

Two different peroxidase systems were tested in this model of yeast biofilm. Firstly, a hydrogen peroxide $\left(\mathrm{H}_{2} \mathrm{O}_{2}\right) / \mathrm{LP}(1 \mathrm{U} / \mathrm{mL}) /$ iodide ( $\left.\mathrm{I}^{-}, 1.2 \mathrm{mM}\right)$ system generating hypoiodite $\left(\mathrm{OI}^{-}\right)$and secondly a $\mathrm{H}_{2} \mathrm{O}_{2} / \mathrm{LP}(1 \mathrm{U} / \mathrm{mL}) /$ thiocyanate $\left(\mathrm{SCN}^{-}, 1.2 \mathrm{mM}\right)$ system producing hypothiocyanite $\left(\mathrm{OSCN}^{-}\right) \cdot \mathrm{H}_{2} \mathrm{O}_{2}$ was enzymatically produced by glucose-oxidase (GOD, $1.3 \mathrm{U} / \mathrm{ml}$ or $0.2 \mathrm{U} / \mathrm{mL}$ ) and glucose $(\mathrm{G})$ present in the culture medium $(115 \mathrm{mM})$.

\section{Statistics}

Data were analyzed using the GraphPad Prism version 5.00 (GraphPad Software ${ }^{\circledR}$, San Diego, CA). ANOVA completed by a Dunnett's or Bonferroni's post-test were performed for statistical comparisons using the same software. Mean values were expressed with their related standard error of the mean unless otherwise indicated.

\section{Results \\ Candida albicans growth in planktonic phase and adhesion to tested materials}

The growing yeast phase in supernatant was evaluated by measuring the $600 \mathrm{~nm}$ spectrophotometer absorbance $\left(\mathrm{A}_{600 \mathrm{~nm}}\right)$ after different periods of incubation (2-21 days). After removal of the supernatant, the immersed materials were washed with sterile broth and the turbidity of the subsequent supernatant after sedimentation was monitored using the same method. Figure 2 illustrates the evolution of $\mathrm{A}_{600 \mathrm{~nm}}$ during the washing process of different materials (titanium powder and foil, resin foil) after a two-day incubation in a contaminated environment. When titanium powder was contaminated by a yeast liquid culture $\left(A_{600 \mathrm{~nm}}: 0.653 \pm 0.074, \mathrm{n}=21\right)$ just before the washing process, the sampling of the supernatant after a 10-minute sedimentation enabled the recovery of only $86.7 \% \pm 2.9 \%$ of the initial absorbance at $600 \mathrm{~nm}(\mathrm{n}=16)$. This discrepancy (-13.3\%) from the theoretic value of $100 \%$ is statistically significant (one-sample t-test, $P=0.0004$ ). The three subsequent washing-mixing-sedimentation cycles decreased supernatant turbidity to $7.0 \% \pm 1.0 \%$ of the initial value $(\mathrm{n}=18)$ according to a one-phase exponential decay model ( $\left.\mathrm{y}=100 \mathrm{e}^{-1.26 \mathrm{x}}, r=0.9940\right)$.

By contrast, when titanium powder was incubated for two days with yeast cells, the liquid-phase turbidity of the third washing in the assays still yielded $110.1 \% \pm 15.0 \%$ of 


\section{A. Titanium powder}

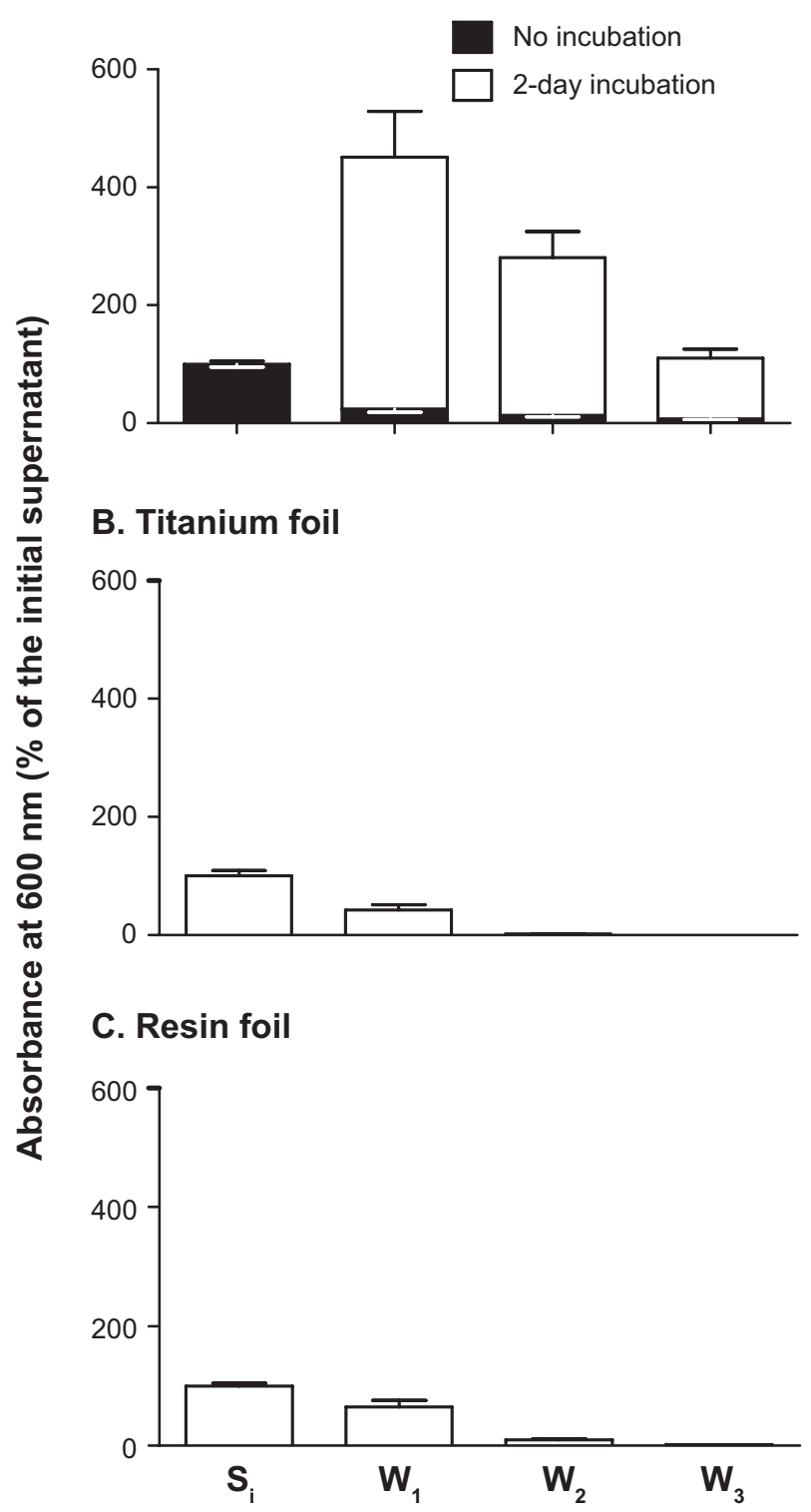

Figure 2 Washing process evaluation for different materials (titanium powder and foil, resin foil) after a two-day incubation in a contaminated environment. $S_{i}$ : first supernatant after a two-day incubation; $\mathrm{W}_{1-3}$ : supernatant after each of the three washing steps.

Note: After incubation (two days), the immersed materials (titanium powder, titanium, and resin foil) were washed three times $\left(\mathrm{W}_{1-3}\right)$ with sterile broth. The turbidity of the subsequent supernatant after sedimentation was monitored at $600 \mathrm{~nm}$. Full columns represent assays where titanium powder has been washed immediately after contamination by a yeast liquid culture. Data are expressed as the percentage of the absorbance (A $600 \mathrm{~nm}$ ) measured in the supernatant after a two-day incubation.

the initial value $(n=26)$. Surprisingly, in the first and second washing of titanium powder, the turbidity of the broth increased to $451.2 \% \pm 77.7 \%(n=25)$ and $280.7 \% \pm 44.1 \%(n=30)$, suggesting desorption from an anchored phase; the decay of turbidity from the highest value was found to be linear $(r=0.9999)$. Similar experiments conducted with titanium and resin foils also showed a linear decrease of turbidity in the liquid broth used for the washing solution $(\mathrm{y}=-49 \mathrm{x}+97$, $r=0.9948$ and $\mathrm{y}=-45 \mathrm{x}+1030, r=0.9919$, respectively) rather than a one-phase exponential decay. In the case of titanium foils, absorbance at $600 \mathrm{~nm}$ yielded $42.3 \% \pm 8.6 \%$ $(\mathrm{n}=12)$ and $2.0 \% \pm 0.5 \%(\mathrm{n}=12)$ of the initial value in the first and second washing medium, respectively. In the case of the resin foils, it yielded $64.8 \% \pm 11.1 \%(n=24)$ and $9.5 \% \pm 2.0 \%(n=24)$ of the initial value in the first and second washing medium, respectively. When contaminated titanium powder was washed immediately, the anchored blastoconidia amount was evaluated as $1.1 \pm 0.1 \times 10^{6}$ blastoconidia per $\mathrm{g}$ of titanium powder.

As shown in Table 1, the MTT-formazan assay conducted in the titanium powder phase after a two-day incubation and three washings showed the presence of a residual adherent biomass equivalent to $7.5 \pm 0.6 \times 10^{6}$ blastoconidia $(n=30)$ per $g$ of titanium powder instead of $494.9 \pm 40.2 \times 10^{3}$ blastoconidia per $\mathrm{cm}^{2}(\mathrm{n}=12)$ on titanium foil $(13.9 \pm 0.2 \mathrm{mg}$, $\mathrm{n}=12$ ). Moreover, the presence of anchored Candida biofilm was also demonstrated on two different resins (hard and soft, used for denture manufacturing) and Falcon tube polystyrene wall. This amount represented $20.5 \pm 3.9 \times 10^{3} / \mathrm{cm}^{2}(\mathrm{n}=12)$, $13.7 \pm 3.2 \times 10^{3} / \mathrm{cm}^{2}(\mathrm{n}=12)$ and $36.7 \pm 3.7 \times 10^{3} / \mathrm{cm}^{2}(\mathrm{n}=4)$ for soft resin, hard resin, and polystyrene assay tube wall, respectively. Microscopic examination confirmed the presence of yeast on the surface of titanium powder.

Figure 3 demonstrates different morphologic aspects of Candida biofilm onto titanium. Figure 3 a shows one titanium grain surrounded by a layer of blastoconidia (magnification $\times 400)$ after one week incubation and three washes. After a longer incubation (three weeks), filamentous structures (hyphae and pseudohyphae) can be observed (Figure 3b). The lower panel shows titanium particles after three weeks' incubation and four washings. In this condition, some blastoconidia were still present with a trabecular structure (Figure 3c) which can be stained by the fluorescent Brightener 28, a polysaccharide structure marker for fluorescent light.

\section{Time course of Candida biofilm production on titanium powder}

Development of yeast biomass was monitored weekly for three weeks in the planktonic phase and in the anchored phase by the procedures mentioned above. Figure 4 (upper panel) reports the time course of the supernatant absorbance at $600 \mathrm{~nm}$, reflecting the planktonic yeast mass. Turbidity reached $0.653 \pm 0.074$ $(n=21)$ after a two-day incubation at room temperature; then it slightly increased (to $0.781 \pm 0.095, \mathrm{n}=4$, on day 7 ; and to $0.929 \pm 0.142, n=4$, on day 14 ). After the second week, it 
Table I Number of blastoconidia anchored onto different materials (titanium, resin, and polystyrene)*

\begin{tabular}{|c|c|c|}
\hline Material & Incubation & Adherent blastoconidia number \\
\hline Powder & & Number/g \\
\hline \multirow[t]{2}{*}{ Titanium } & $\begin{array}{l}\text { Immediate } \\
\text { treatment }\end{array}$ & $1.1 \pm 0.1 \times 10^{6} / g(n=3)$ \\
\hline & Two-day & $7.5 \pm 0.6 \times 10^{6} / g(n=30)$ \\
\hline Foil & & Number/cm² \\
\hline Titanium & Two-day & $\begin{array}{l}494.9 \pm 40.2 \times 10^{3} / \mathrm{cm}^{2}(\mathrm{n}=12 \text { ) } \\
\text { (equivalent to } 35.6 \pm 2.9 \times 10^{6} / \mathrm{g} \text { ) }\end{array}$ \\
\hline Soft resin & Two-day & $20.5 \pm 3.9 \times 10^{3} / \mathrm{cm}^{2}(\mathrm{n}=12)$ \\
\hline Hard resin & Two-day & $13.7 \pm 3.2 \times 10^{3} / \mathrm{cm}^{2}(\mathrm{n}=12)$ \\
\hline Polystyrene & Two-day & $36.7 \pm 3.7 \times 10^{3} / \mathrm{cm}^{2}(\mathrm{n}=4)$ \\
\hline
\end{tabular}

*Incubation was at room temperature. Material-adherent blastoconidia were evaluated by the MTT procedure. Data are expressed per mass unit for powder or per surface unit for foils.

decreased and reached $0.161 \pm 0.058(n=4)$ on day 21 , which is less than the values previously obtained (corresponding to $24.7 \% \pm 9.3 \%$ of those found on day 2 and $17.3 \% \pm 6.8 \%$ of those on day 14). An ANOVA test completed by a Bonferroni's multiple comparison post-test did not show any difference between days 2,7 , and $14(P>0.5)$; but the turbidity on day 21 was significantly decreased in comparison with values obtained earlier (ANOVA, $P=0.0078$ ). The time course of the anchored yeast mass was rather different (Figure 4, lower panel), reaching steady state on day $2(0.924 \pm 0.060, \mathrm{n}=30)$ and persisting until day $21(0.887 \pm 0.044, \mathrm{n}=4)$. Intermediate values were $0.829 \pm 0.206(\mathrm{n}=4)$ on day 7 and $1.280 \pm 0.112$ $(n=4)$ on day 14 . An ANOVA test completed by a Bonferroni's multiple comparison post-test did not show any difference between the anchored yeast mass obtained for different periods of incubation (ANOVA, $P=0.1717$ ).

\section{Modulation of Candida biofilm production on titanium powder by peroxidase systems}

In the presence of $1.3 \mathrm{U} / \mathrm{mL}$ GOD and $115 \mathrm{mM} \mathrm{G}$, both $\mathrm{G} / \mathrm{GOD} / \mathrm{I}^{-} / \mathrm{LP}$ and $\mathrm{G} / \mathrm{GOD} / \mathrm{SCN}^{-} / \mathrm{LP}$ systems prevented Candida growth in the planktonic as well as the attached phase for at least 21 days. Table 2 indicated the absorbance values at $600 \mathrm{~nm}$ (for the planktonic evaluation) and at $570 \mathrm{~nm}$ (for the attached phase evaluation) expressed as the percentage of the time-paired control in the absence of oxidant. Both peroxidase systems maintained the titanium powder free of yeast cells for three weeks, with absorbance at $600 \mathrm{~nm}$ equal to zero. Repeated measurements $(n=4)$ at one, two, and three weeks showed persisting growth inhibition in the broth. By contrast, a limited but existing anchored phase was demonstrated on titanium powder by the MTT procedure.
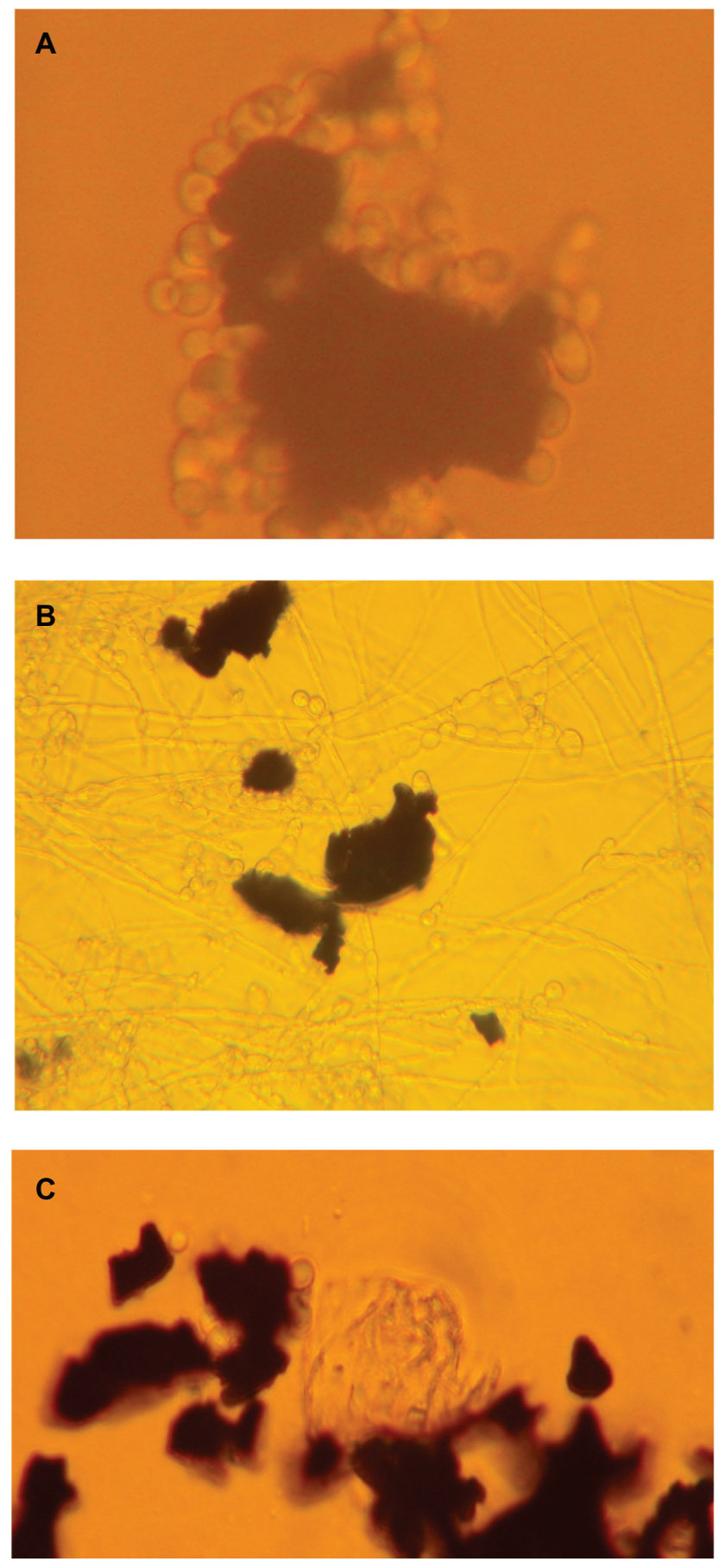

Figure 3 A) Candida albicans blastoconidia adherent to titanium granule (magnification X400 in the absence of any stain procedure) after a one-week incubation and three washings. B) Titanium grains surrounded by filamentous structures (hyphae and pseudohyphae) after a three-week incubation. C) Titanium particles (after three weeks of incubation and four washings) with some remaining blastoconidia and trabecular structure.

In the presence of the $\mathrm{SCN}^{-}$substrate $(\mathrm{n}=4)$, a small anchored phase was observed to evolve from $11.3 \% \pm 12.3 \%$, to $24.9 \% \pm 8.3 \%$, then finally to $18.7 \% \pm 3.5 \%$ of the control on days 7,14 , and 21 , respectively. In the presence of $\mathrm{I}^{-}$substrate $(n=4)$, no anchored biomass could be shown (zero 


\section{A. Planktonic phase}

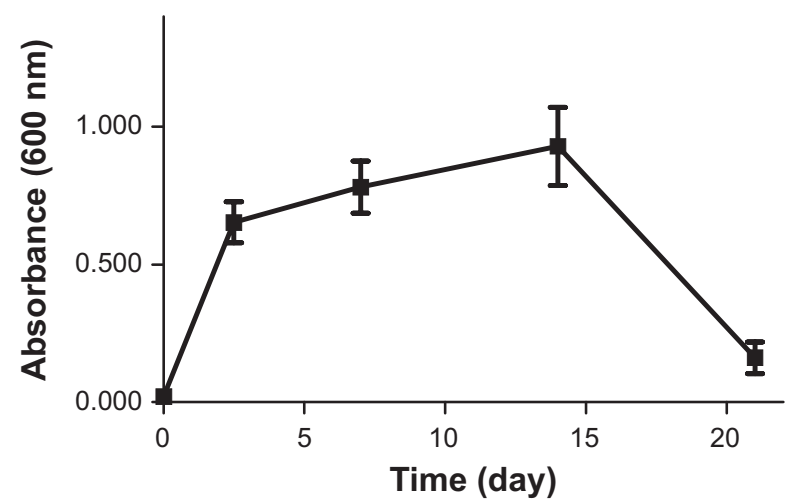

\section{B. Anchored phase}

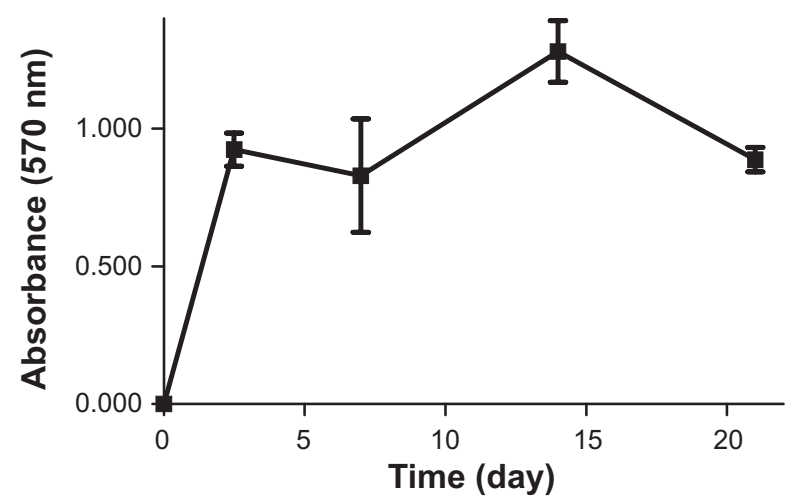

Figure 4 Time course of yeast biomass in the planktonic phase (top) and in the anchored phase (bottom).

Note: Development of yeast biomass was weekly monitored for three weeks in the planktonic phase (absorbance at $600 \mathrm{~nm}$ ) and in the anchored phase (absorbance at $570 \mathrm{~nm}$ after MTT procedure).

absorbance at $570 \mathrm{~nm}$ ) after one week of incubation at room temperature, and only appeared after 14 days' incubation $(14.5 \% \pm 3.7 \%$ of the paired control) and slightly progressed to $20.2 \% \pm 3.7 \%$ of the control on day 21 . At any incubation time, data from both peroxidase system conditions were statistically lower than their paired controls (ANOVA completed by a Dunnett's multiple comparison test).

\section{Yeast recovery from deposits onto titanium foil}

Droplets $(20 \mu \mathrm{L}$ of sterile water) containing small amounts of yeast (10-200 blastoconidia in five independent experiments) were deposited onto titanium foil and incubated at $37^{\circ} \mathrm{C}$ for 30 minutes. Foils were then rubbed on Sabouraud solid medium for cultures at $37^{\circ} \mathrm{C}$ for two days. Yeast recovery $(81.9 \% \pm 7.0 \%, \mathrm{n}=5)$ was statistically independent (ANOVA, $\mathrm{P}=0.6167$ ) of the numbers of blastoconidia deposited onto the foil. Figure 4 reports Candida recovery after rubbing the foil prepared in the following conditions: a) blastoconidia droplets were directly deposited onto foil, b) blastoconidia droplets

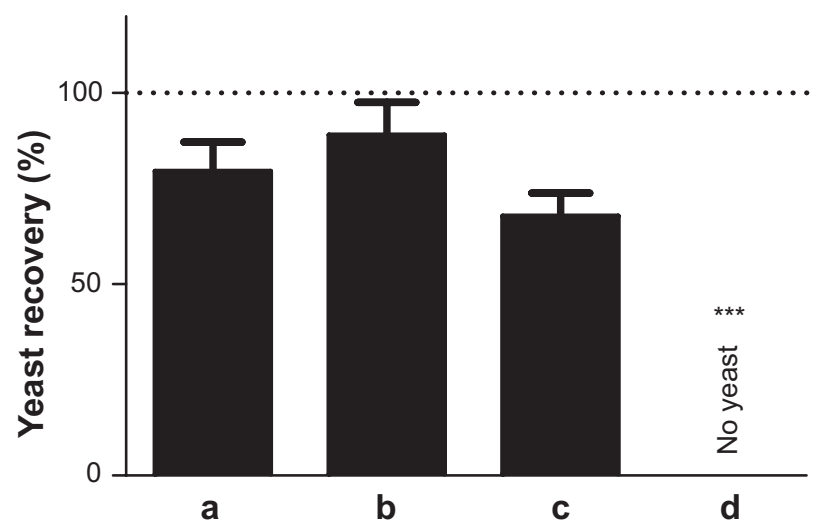

Figure 5 Yeast recovery from deposits onto titanium foil.

Note: Droplets $(20 \mu \mathrm{L}$ of sterile water containing from 10 to 200 blastoconidia) were deposited onto titanium foil and incubated at $37^{\circ} \mathrm{C}$ for 30 minutes. Candida albicans recovery was evaluated after rubbing the foil prepared in the following conditions: a) blastoconidia droplets were directly deposited onto foil; b) blastoconidia droplets were deposited onto lactoperoxidase-coated titanium; and c) blastoconidia droplets with added G/GOD were deposited onto lactoperoxidase-coated titanium, d) blastoconidia droplets with added G/GOD/KI were deposited onto lactoperoxidasecoated titanium ( $n=5$ for each condition). Data were analyzed by an ANOVA test $(P=0.0009)$ completed by a Dunnett's multiple comparison test $\left({ }^{50 k} P<0.00 \mathrm{I}\right)$. Abbreviations: G, glucose; GOD, glucose oxidase; LP, lactoperoxidase; $\mathrm{I}^{-}$, iodide; $\mathrm{SCN}^{-}$, thiocyanate.

were deposited onto LP-coated titanium, c) blastoconidia droplets with added G/GOD were deposited onto LP-coated titanium, d) blastoconidia droplets with added G/GOD/KI were deposited onto LP-coated titanium. Yeast recovery was respectively $79.5 \% \pm 7.7 \%(n=6), 89.0 \% \pm 8.6 \%(n=4)$, $67.8 \% \pm 6.0 \%(n=6)$, and $0.0 \% \pm 0.0 \%(n=6)$. LP coating only operated when the droplets contained the three other components $(\mathrm{G} / \mathrm{GOD} / \mathrm{KI})$ of the system, producing OI. An ANOVA test $(P=0.0009)$ completed by a Dunnett's multiple comparison test $(P<0.001)$ was then highly significant.

\section{Discussion}

Frequent occurrence of C. albicans at peri-implantitis sites, ${ }^{7}$ inside port reservoirs of totally implantable vascular access devices, ${ }^{1}$ and other titanium implants justifies laboratory attempts to devise a reproducible model in order to investigate in vitro yeast biofilm formation and its modulation. This paper shows the reliability of a model using titanium powder and foil as support for C. albicans adhesion and biofilm formation with the aim to study yeast growth inhibitors on biomaterial surfaces, in particular titanium implants. Numerous in vitro biofilm models have already been described on various material surfaces in order to investigate new antimicrobial strategies. However, only a few studies have evaluated titanium support in the yeast biofilm area. One paper $^{15}$ reported the efficiency of a laser decontamination procedure in dentistry applied to a titanium model derived from previous studies ${ }^{19}$ of yeast biofilms on denture polymethylmethacrylate surfaces. 
Table 2 Time course of yeast biomass in the planktonic phase and in the anchored phase

\begin{tabular}{|c|c|c|c|c|}
\hline \multicolumn{5}{|c|}{ Planktonic phase evaluated by turbidimetry } \\
\hline Incubation time (days) & Control A (600 nm) & Control (\%) & G/GOD/SCN-/LP (\%) & G/GOD/I-/LP (\%) \\
\hline 7 & $0.78 \mathrm{I} \pm 0.095(4)$ & $100.0 \pm 17.2 .0(4)$ & $0.0 \pm 0.1(4)^{* * *}$ & $0.0 \pm 0.3(4)^{* * *}$ \\
\hline 14 & $0.929 \pm 0.142(4)$ & $100.0 \pm 21.6(4)$ & $0.0 \pm 0.0(4)^{* * *}$ & $0.0 \pm 0.0(4)^{* * *}$ \\
\hline 21 & $0.161 \pm 0.058(4)$ & $100.0 \pm 50.9(4)$ & $0.0 \pm 0.0(4)$ & $0.0 \pm 0.0(4)$ \\
\hline \multicolumn{5}{|c|}{ Anchored phase evaluated by MTT assay } \\
\hline Incubation time (days) & Control A (570 nm) & Control (\%) & G/GOD/SCN-/LP (\%) & G/GOD/I-/LP (\%) \\
\hline 7 & $0.829 \pm 0.206(4)$ & $100.0 \pm 35.1(4)$ & $11.3 \pm 12.3(4)^{*}$ & $0.0 \pm 0.0(4)^{*}$ \\
\hline 14 & $1.280 \pm 0.112(4)$ & $100.0 \pm 12.4(4)$ & $24.9 \pm 8.3(4)^{* * *}$ & $14.5 \pm 3.7(4)^{* * *}$ \\
\hline 21 & $0.887 \pm 0.044(4)$ & $100.0 \pm 7.0(4)$ & $18.7 \pm 3.5(4)^{* * *}$ & $20.2 \pm 3.7(4)^{* * * *}$ \\
\hline
\end{tabular}

Notes: Incubation at room temperature for 7-2I days in the presence of peroxidase systems with either thiocyanate $\mathrm{SCN}^{-}$or $\mathrm{I}^{-}$substrate. Data are expressed as the percentage of the time-paired control without the peroxidase system. Data from both peroxidase system conditions were compared to the corresponding control condition by ANOVA completed by a Dunnett's multiple comparison test (*P $<0.05$; **P $<0.0$ I; $* * * P<0.00 \mathrm{I}$ ).

Abbreviations: G, glucose; GOD, glucose oxidase; LP, lactoperoxidase; $\mathrm{I}^{-}$, iodide; $\mathrm{SCN}^{-}$, thiocyanate.

The present study uses similar inoculum sizes and procedures for the quantification of yeast biomass anchored to a titanium support, as in the two last quoted papers. Nevertheless, the present model differs in several aspects, ie, the use of material powder increases support surfaces in the same manner as cell cultures on beads. Moreover, adhesion of $C$. albicans to the support precedes the formation of a biofilm. These two steps are not set apart in the model described here, which enables the simultaneous study of yeast growth both in the supernatant (planktonic phase) and in the biofilm (anchored phase). Within a very short time, anchored blastoconidia progressively covered the available surface on the biomaterial. The small but significant decrease of culture liquid turbidity immediately after addition of titanium powder should reflect attachment of Candida to titanium, starting as early as the material has been immersed. The anchored blastoconidia amount was then evaluated by using the MTT method as $1.1 \pm 0.1 \times 10^{6}$ blastoconidia per $\mathrm{g}$ of titanium powder, demonstrating a rapid attachment. After covering the entire available surface, biofilm grew following a multilayer pattern (as seen in Figure 3a), susceptible to liberation of numerous blastoconidia during the washing process. This desorption resulted in modifying the decay model of turbidity during the washings. After the longest incubation (three weeks), titanium powder was observed embedded in a network of hyphae. To investigate the time course of events, planktonic and biofilm growths were evaluated between two days and three weeks. As shown in the results section, absorbance at $600 \mathrm{~nm}$ of the liquid medium rapidly increased to reach a steady state at two days, then decreased after two weeks, in agreement with theoretic microbiologic growth curves, the decline being attributed to modifications of the culture liquid (eg, nutrient exhaust, toxic catabolites, and $\mathrm{pH}$ change). MTT reduction assay data revealed that the $C$. albicans biofilm developed rapidly (at least in two days), while the growth decline stage was delayed (albeit this was not part of this investigation). Photonic and fluorescent microscopy was performed at all stages in order to visualize the biofilm structure, composed of a dense blastoconidia layer and hyphal forms. At 21 days, added extracellular polymeric material could be observed, with an amorphous reticular appearance. Initially, C. albicans cells were observed as blastoconidia, and (pseudo)hyphae appeared later. Production of extracellular polysaccharide material by Candida cells and filamentation of yeast cells contribute to modify biofilm architecture into a heterogeneous structure. Further studies should describe more precisely the kinetics of Candida biofilm formation on titanium.

Titanium is widely employed for implant manufacturing due to its good biocompatibility and mechanical properties, but infection remains a cause of failure leading to removal. The titanium surface is not antimicrobial by itself, so it could be used as support for a Candida biofilm in this investigation. Making implant surfaces resistant to microbial colonization should reduce infectious complications. Such developments need an in vitro model which allows investigation of the effect of surface modification and coatings on biofilm production. Yeast biofilms are characterized by a significantly decreased susceptibility to usual antifungal drugs, encouraging the search for other inhibition methods, such as peroxidase systems. Previous studies have shown that a 30-minute exposure to $\mathrm{OI}^{-}$was sufficient to inhibit planktonic growth in vitro. ${ }^{14}$ The present investigation demonstrated that Candida biofilm development on material surfaces could be reduced or even suppressed by LP-generated $\mathrm{OI}^{-}$and $\mathrm{OSCN}^{-}$, not only when peroxidase and substrates system were dissolved in the liquid phase into material and immersed, but also when material-precoated peroxidase was activated by simple addition of the substrates to the liquid surrounding the precoated material. Those data demonstrate 
the efficiency of peroxidase systems against a Candida strain, and concomitantly show an application of the titanium powder model described supra. This investigation did not demonstrate that enzymes remain active after a three-week incubation, but clearly showed that their inhibition effect persisted during this time. Previous investigations demonstrated, firstly, that coating onto titanium did not modify LP activity, and secondly, that LP incorporated into oral gel maintained its activity for at least one year and thirdly, that the substrate exhaust (namely $\mathrm{H}_{2} \mathrm{O}_{2}, \mathrm{I}^{-}$) is the actual limiting factor. ${ }^{13,20,21}$ Transfer of this finding from bench to clinic is questionable. Indeed, immunogenicity of coated LP should limit the applications of this system to ex vivo conditions. A previous investigation indicated an antibacterial effect with Gram-positive and Gram-negative bacteria, suggesting a nonspecific inhibitory effect of hypoiodite on microbial metabolism and growth. ${ }^{22}$

\section{Conclusion}

In conclusion, $C$. albicans biofilms on titanium powder could offer a simple and reliable model for further investigating new antimicrobial strategies. Moreover, the model could be used in other studies of contaminating microorganisms on implanted materials.

\section{Acknowledgments}

The authors thank F Chagri and J Le Gentil de Rosmorduc from the Haute Ecole Francisco Ferrer (Medical Biology section) for their enthusiastic participation in this project. The authors also thank Pr C de Burbure for her review of the manuscript, and Pr M Stas and Pr L Sennerby for their helpful advice.

\section{Disclosure}

The authors report no conflicts of interest in this work.

\section{References}

1. Brouns F, Schuermans A, Verhaegen J, De Wever I, Stas M. Infection assessment of totally implanted long-term venous access devices. J Vasc Access. 2006;7:24-28.

2. Chang L, Tsai JS, Huang SJ, Shih CC. Evaluation of infectious complications of the implantable venous access system in a general oncologic population. Am J Infect Control. 2003;31:34-39.

3. Opilla M. Epidemiology of bloodstream infection associated with parenteral nutrition. Am J Infect Control. 2008;36:S173.e5-e8.

4. Holzheimer RG, Dralle H. Management of mycoses in surgical patients - review of the literature. Eur J Med Res. 2002;7:200-206.

Medical Devices: Evidence and Research

\section{Publish your work in this journal}

Medical Devices: Evidence and Research is an international, peerreviewed, open access journal that focuses on the evidence, technology, research, and expert opinion supporting the use and application of medical devices in the diagnosis, treatment and management of clinical conditions and physiological processes. The identification of novel
5. Mermel LA, Allon M, Bouza E, et al. Clinical practice guidelines for the diagnosis and management of intravascular catheter-related infection: 2009 Update by the Infectious Diseases Society of America. Clin Infect Dis. 2009;49:1-45.

6. Penk A, Pittrow L. Role of fluconazole in the long-term suppressive therapy of fungal infections in patients with artificial implants. Mycoses. 1999;42(S2):91-96.

7. Leonhardt A, Renvert S, Dahlén G. Microbial findings at failing implants. Clin Oral Implants Res. 1999;10:339-345.

8. Shibli JA, Martins MC, Lotufo RF, Marcantonio E Jr. Microbiologic and radiographic analysis of ligature-induced peri-implantitis with different dental implant surfaces. Int J Oral Maxillofac Implants. 2003;18:383-390.

9. Arweiler-Harbeck D, Sanders A, Held M, Jerman M, Ehrich H, Jahnke K. Does metal coating improve the durability of silicone voice prostheses? Acta Otolaryngol. 2001;121:643-646.

10. Joshi RI, Eley A. The in-vitro effect of a titanium implant on oral microflora: Comparison with other metallic compounds. J Med Microbiol. 1988;27:105-107.

11. Akiba N, Hayakawa I, Keh ES, Watanabe A. Antifungal effects of a tissue conditioner coating agent with $\mathrm{TiO}_{2}$ photocatalyst. $J$ Med Dent Sci. 2005;52:223-227.

12. Darouiche RO, Green G, Mansouri MD. Antimicrobial activity of antiseptic-coated orthopaedic devices. Int J Antimicrob Agents. 1998; 10:83-86.

13. Ahariz M, Mouhyi J, Louette P, Van Reck J, Malevez C, Courtois P. Adsorption of peroxidase on titanium surfaces: A pilot study. J Biomed Mater Res. 2000;52:567-571.

14. Majerus P, Courtois P. Susceptibility of Candida albicans to peroxidasecatalyzed oxidation products of thiocyanate, iodide and bromide. J Biol Buccale. 1992;20:241-245.

15. Sennhenn-Kirchner S, Schwarz P, Schliephake H, Konietschke F, BrunnerE, Borg-vonZepelin M.Decontamination efficacy of erbium:yttriumaluminium-garnet and diode laser light on oral Candida albicans isolates of a 5-day in vitro biofilm model. Lasers Med Sci. 2009;24:313-320.

16. Liu LF, Barford J, Yeung KL, Si G. Non-UV based germicidal activity of metal-doped $\mathrm{TiO}_{2}$ coating on solid surfaces. J Environ Sci. 2007;19:745-750.

17. Levitz SM, Diamond RD. A rapid colorimetric assay of fungal viability with the tetrazolium salt MTT. J Infect Dis. 1985;152:938-945.

18. Basivireddy J, Jacob M, Balasubramanian KA. Oral glutamine attenuates indomethacin-induced small intestinal damage. Clin Sci. 2004;107:281-289.

19. Chandra J, Mukherjee PK, Leidich SD, et al. Antifungal resistance of candidal biofilms formed on denture acrylic in vitro. $J$ Dent Res. 2001;80:903-908.

20. Vanden Abbeele A, de Meel H, Ahariz M, Perraudin J-P, Beyer I, Courtois P. Denture contamination by yeasts in the elderly. Gerodontology. 2008;25:222-228.

21. Ahariz M, Courtois P. Candida albicans susceptibility to lactoperoxidase-generated hypoiodite. Clinical, Cosmetic and Investigational Dentistry. 2010;2: In Press.

22. Courtois P, Vanden Abbeele A, Amrani N, Pourtois M. Streptococcus sanguis survival rates in the presence of lactoperoxidase-produced $\mathrm{OSCN}^{-}$and $\mathrm{OI}^{-}$. Med Sci Res. 1995;23:195-197.

\section{Dovepress}

devices and optimal use of existing devices which will lead to improved clinical outcomes and more effective patient management and safety is a key feature. The manuscript management system is completely online and includes a quick and fair peer-review system. Visit http://www. dovepress.com/testimonials.php to read real quotes from authors. 